\section{Cureus}

Received 07/12/2017

Review began 07/26/2017

Review ended 07/27/2017

Published 08/02/2017

\section{(c) Copyright 2017}

Desai et al. This is an open access article distributed under the terms of the Creative Commons Attribution License CC-BY 3.0., which permits unrestricted use, distribution, and reproduction in any medium, provided the original author and source are credited.

\title{
Impact of Cocaine Use on Acute Ischemic Stroke Patients: Insights from Nationwide Inpatient Sample in the United States
}

Rupak Desai ${ }^{1}$, Upenkumar Patel ${ }^{2}$, Chintan Rupareliya ${ }^{3}$, Sandeep Singh ${ }^{4}$, Manan Shah ${ }^{5}$, Rikinkumar S. Patel ${ }^{6}$, Smit Patel ${ }^{7}$, Zabeen Mahuwala ${ }^{8}$

1. Cardiology, Atlanta Veterans Affairs Medical Center, Decatur, USA 2. Internal Medicine, Nassau University Medical Center, New york, USA 3. Department of Neurology, University of Kentucky College of Medicine 4. Clinical Epidemiology, Biostatistics and Bioinformatics, Academic Medical Center University of Amsterdam, Amsterdam, NLD 5. Internal Medicine, University of Texas, Houston 6. Psychiatry, Griffin Memorial Hospital, Norman, USA 7. Department of Neurology, University of Connecticut Health Center 8. Department of Neurology, University of Kentucky College of Medicine, Lexington, USA

$\square$ Corresponding author: Chintan Rupareliya, drchintan2000@hotmail.com Disclosures can be found in Additional Information at the end of the article

\section{Abstract}

Cocaine is the third most common substance of abuse after cannabis and alcohol. The use of cocaine as an illicit substance is implicated as a causative factor for multisystem derangements ranging from an acute crisis to chronic complications. Vasospasm is the proposed mechanism behind adverse events resulting from cocaine abuse, acute ischemic strokes (AIS) being one of the few. Our study looked into in-hospital outcomes owing to cocaine use in the large population based study of AIS patients. Using the national inpatient sample (NIS) database from 2014 of United States of America, we identified AIS patients with cocaine use using International Classification of Disease, Ninth Revision (ICD-9) codes. We compared demographics, mortality, in-hospital outcomes and comorbidities between AIS with cocaine use cohort versus AIS without cocaine use cohort. Acute ischemic strokes (AIS) with cocaine group consisted of higher number of older patients (> 85 years) $(25.6 \%$ versus $18.7 \%, \mathrm{p}<0.001$ ) and females $(52.4 \%$ versus $51.0 \%, \mathrm{p}<0.001)$. Cocaine cohort had higher incidence of valvular disorders (13.2\% versus $9.7 \%, \mathrm{p}<0.001)$, venous thromboembolism $(3.5 \%$ versus $2.6 \%, \mathrm{p}<0.03$ ), vasculitis $(0.9 \%$ versus $0.4 \%, \mathrm{p}<0.003)$, sudden cardiac death $(0.4 \%$ versus $0.2 \%, \mathrm{p}<0.02)$, epilepsy (10.1\% versus $7.4 \%, \mathrm{p}<0.001)$ and major depression $(13.2 \%$ versus $10.7 \%, \mathrm{p}<0.007)$. The multivariate logistic regression analysis found cocaine use to be the major risk factor for hospitalization in AIS cohort. In-hospital mortality (odds ratio $(\mathrm{OR})=1.4,95 \%$ confidence interval=1.1-1.9, $\mathrm{p}<0.003$ ) and the disposition to short-term hospitals (odds ratio $(\mathrm{OR})=2.6$, $95 \%$ confidence interval $=2.1-3.3, \mathrm{p}<0.001$ ) were also higher in cocaine cohort. Venous thromboembolism was observed to be linked with cocaine use $(\mathrm{OR}=1.5,95 \%$ confidence interval $=1.0-2.1, \mathrm{p}<0.01)$ but less severely than vasculitis $(\mathrm{OR}=3.0,95 \%$ confidence interval $=$ $1.6-5.8, \mathrm{p}<0.001)$. Further prospective research is warranted in this direction to improve the outcomes for AIS and lessen the financial burden on the healthcare system of the United States.

Categories: Neurology, Psychiatry, Quality Improvement Keywords: cocaine abuse, acute ischemic stroke, cocaine dependence, stroke prevention, in-hospital outcomes, national inpatient sample, mortality 


\section{Introduction}

The use of cocaine as an illicit drug surged in the United States of America between 2002 and 2007 and currently, it is the second most abused drug after cannabis and alcohol [1-3]. Among the adults, the annual prevalence of cocaine use was $1.5 \%$, while some states reported the prevalence of $5.5 \%$ to $5.8 \%$ in the age group of 18 to 25 years. The United Nations Office on Drugs and Crime and the European Monitoring Centre for Drugs and Drug Addiction has reported an increase of cocaine use in particular parts of the world [4]. The burden of health care due to cocaine dependence was reported high in the recent studies [4]. Around 23.9 million people aged 12 years and above were reported using illicit drugs in 2012 according to data from the National Survey on Drug Use and Health [5]. A recent study from a community hospital found $2.3 \%$ people being cocaine positive during drug screening in the age group of 65 years and older. In the USA, the areas of primary concern are the one rampaged with poverty and poor education [6]

One report from the emergency department notes in the Detroit area in 2002 showed cocaine use of around 182/100,000 of the population [7]. It is not merely a problem of one country; rather it has turned into a global issue [1]. The cocaine use leads to the spectrum of multisystem derangements ranging from mild intoxication to severe complications like acute myocardial infarction, seizures and acute ischemic stroke [8]. Compared to the corresponding peer groups in the general population, cocaine users tend to have four to eight times higher mortality [9-10]. Use of cocaine is presumed to be one of the major risk factors for cerebrovascular disease, including stroke. Acute ischemic stroke (AIS) is labeled as the third leading cause of disability-adjusted life years [10].

Previous studies have reported a 19\% increase in the incidence of strokes due to cocaine use in the last two decades [11]. The rise in cocaine-associated morbidity and mortality posed it as a major public health concern [11-12]. Impacts on health care economies due to stroke-related disability is devastating owing to medical cost, rehabilitation cost and cost due to loss of workforce. A direct or indirect burden of around $\$ 68.9$ billion was imposed on the US healthcare owing to strokes in 2009, a major part of which was comprised of strokes as a result of illicit drugs use [2]. We aim to evaluate various factors associated with acute ischemic strokes (AIS) risk and to develop the management strategies to mitigate mortality rates within cocaineinduced stroke population.

\section{Materials And Methods}

\section{Data source}

We utilized the discharge data from the National Inpatient Sample (NIS) of the Healthcare Cost and Utilization Project (HCUP) as a source. The NIS is an all-payer dataset that includes around eight million (around 20\% of the stratified sample) inpatient admissions and discharges from almost 1050 USA hospitals, excluding long term care and rehabilitation facilities. The NIS data set is unweighted and it results in the weighted estimate of the total discharge number of the US population when we apply the discharge weight to the unweighted data. We excluded the data of missing information such as age, gender, discharge condition or primary diagnosis. We used the International Classification of Diseases, Ninth Revision, Clinical Modification (ICD-9$\mathrm{CM}$ ) to identify admissions with the primary diagnosis of AIS. Since NIS is publicly available deidentified database from Agency for Healthcare Research and Quality (AHRQ), it does not require an approval from institutional review board (IRB). More details on the dataset content and methods of collections are accessible on the HCUP website [13].

\section{Patient selection}

We looked into the NIS database of the year 2014 to identify all patients with AIS with ICD-9- 


\section{Cureus}

CM codes (433.01, 433.11, 433.21, 433.31, 433.81, 433.91, 434.01, 434.11, 434.91 and 436). Current use of cocaine (dependence or abuse) was identified using the ICD-9-CM codes 304.20 (cocaine dependence, unspecified), 305.62 (non-dependent cocaine use, episodic), 305.61 (nondependent cocaine use, continuous), 304.21 (cocaine dependence, continuous) and 304.22 (cocaine dependence, episodic) [14] (Appendix).

\section{Variables}

Demographic variables examined in this study included age group (1-17, 18-44, 45-64, 65-84 years and > 85), gender (male or female), race (white, Hispanic, Asian or Pacific Islander, Native American and other) and payer source (Medicare, Medicaid, private pay, self-pay, no charge and other). Based on existing literature, we searched and defined AIS risk factors using diagnosis codes from ICD-9-CM mentioned in the appendix.

\section{Statistical analysis}

Statistical Package for the Social Science, version 22.0 (SPSS V.22.0, IBM Corp., Armonk, NY, USA) was used for all the statistical analysis. The incidence of AIS hospitalization among cocaine users and nonusers were determined by searching all available diagnosis fields for the diagnosis of AIS. Further age stratification was performed of the population into groups of 1-17, 18-44, 45-64, 65-84 years and > 85 years. Pearson's chi-square test was used for categorical data and the independent sample T-test was used for continuous data. We used a multivariate logistic regression model to assess the AIS outcomes of cocaine use. Standard weights from HCUP were utilized to get the national weighted estimates of inpatient admissions. We defined $\mathrm{p}$-value less than $0.05(\mathrm{p}<0.005)$ as the statistical significance.

\section{Results}

\section{Baseline characteristics of acute ischemic strokes cohort}

We identified the total of 584,115 patients with AIS by using the discharge data from NIS of the year 2014. We further stratified cohorts into a cocaine use $(\mathrm{N}=1135)$ and non-cocaine use $(\mathrm{N}=582980)$. The AIS with cocaine group consisted of higher number of older patients (> 85 years) (25.6\% versus $18.7 \%, \mathrm{p}<0.001)$, females $(52.4 \%$ versus $51.0 \%, \mathrm{p}<0.001)$ and whites $(89.2 \%$ versus $70.3 \%, \mathrm{p}<0.001)$. Cardiovascular incidences that were higher in the cocaine cohort versus non-cocaine cohort included valvular disorders $(13.2 \%$ versus $9.7 \%, \mathrm{p}<0.001)$, venous thromboembolism $(3.5 \%$ versus $2.6 \%, \mathrm{p}<0.03)$, vasculitis $(0.9 \%$ versus $0.4 \%, \mathrm{p}<0.003)$, and sudden cardiac death $(0.4 \%$ versus $0.2 \%, \mathrm{p}<0.02)$. The incidence of epilepsy was higher in cocaine cohort $(10.1 \%$ versus $7.4 \%, \mathrm{p}<0.001)$. The incidence of major depression was higher in cocaine cohort $(13.2 \%$ versus $10.7 \%, \mathrm{p}<0.007)$. The incidences of other risk factors for AIS such as atherosclerosis, cardiac circulatory anomalies, hypertension (complicated and uncomplicated), elevated cholesterol, diabetes, transient ischemic attack (TIA), paralysis, family history of stroke, deficiency anemia, coagulopathy, disorders of fluid and electrolytes were significantly higher in the non-cocaine cohort (Table 1).

Variables
Unweighted admissions
Weighted admissions
Age in years at admission

Mean Age \pm SD 


\section{Cureus}

$1-17$
$18-44$
$45-64$
$65-84$

$>85$

Died during hospitalization

Did not die

Died

Disposition of Patient

Routine

To Short-term Hospitals

Other (SNF, ICF, Another facility)

Home Health Care

Against Medical Advice (AMA)

Died

Discharged alive, destinations unknown

Elective Vs. Non-elective Admissions

Non-elective

Elective

Indicator of Sex

Male

Female

Primary Expected Payer

Medicare

Medicaid

Private including $\mathrm{HMO}$

Self - Pay

No charge

Other

Race

White
$93.6 \%$

$65.7 \%$

$0.5 \%$
$4.6 \%$
$28.6 \%$
$47.5 \%$

$93.1 \%$

$6.9 \%$

$32.3 \%$

$3.4 \%$

$43.4 \%$

$13.2 \%$

$0.7 \%$

$6.9 \%$

$0.1 \%$

$6.4 \%$

$49.0 \%$

$51.0 \%$

$9.0 \%$

$18.7 \%$

$4.1 \%$

$0.4 \%$

$2.1 \%$

$70.3 \%$
$0.0 \%$

$<0.001$

$<0.001$

$<0.001$

$<0.001$

$<0.001$

$25.6 \%$

$89.9 \%$

$<0.001$

$10.1 \%$

$<0.001$

$22.5 \%$

$<0.001$

$11.3 \%$

$<0.001$

$37.0 \%$

$<0.001$

$18.1 \%$

$<0.001$

$0.9 \%$

$<0.001$

$10.1 \%$

$<0.001$

$0.0 \%$

$<0.001$

$98.2 \%$

$<0.001$

$1.8 \%$

$<0.001$

$47.6 \%$

0.346

$52.4 \%$

0.346

$72.1 \%$

$<0.001$

$9.3 \%$

$<0.001$

$16.4 \%$

$<0.001$

$1.3 \%$

$<0.001$

$0.0 \%$

$<0.001$

$0.9 \%$

$<0.001$

$89.2 \%$

$<0.001$ 


\section{Cureus}

\begin{tabular}{|c|c|c|c|}
\hline Black & $16.8 \%$ & $6.3 \%$ & $<0.001$ \\
\hline Hispanic & $7.3 \%$ & $4.1 \%$ & $<0.001$ \\
\hline Asian or Pacific Islander & $2.4 \%$ & $0.0 \%$ & $<0.001$ \\
\hline Native American & $0.5 \%$ & $0.0 \%$ & $<0.001$ \\
\hline Other & $2.7 \%$ & $0.5 \%$ & $<0.001$ \\
\hline \multicolumn{4}{|l|}{ Co -morbidities } \\
\hline RA/CVD & $2.7 \%$ & $4.0 \%$ & 0.009 \\
\hline Atherosclerosis & $28.7 \%$ & $25.6 \%$ & 0.020 \\
\hline Acute Myocardial Infarction & $4.6 \%$ & $4.8 \%$ & 0.667 \\
\hline Arrhythmia & $0.3 \%$ & $0.4 \%$ & 0.204 \\
\hline Sudden Cardiac Death & $0.2 \%$ & $0.4 \%$ & 0.024 \\
\hline Cardiac Circulatory Anomalies & $3.0 \%$ & $1.3 \%$ & $<0.001$ \\
\hline Heart Valve Disorders & $11.2 \%$ & $14.1 \%$ & 0.002 \\
\hline Vasculitis & $0.4 \%$ & $0.9 \%$ & 0.003 \\
\hline Hypertension & $79.8 \%$ & $72.2 \%$ & $<0.001$ \\
\hline Elevated BP without Hypertension & $0.2 \%$ & $0.0 \%$ & 0.106 \\
\hline Elevated Cholesterol & $54.7 \%$ & $42.3 \%$ & $<0.001$ \\
\hline Venous Thromboembolism & $2.6 \%$ & $3.5 \%$ & 0.039 \\
\hline Viral Infection & $0.6 \%$ & $0.0 \%$ & 0.007 \\
\hline Pulmonary Circulation Disorders & $4.1 \%$ & $6.2 \%$ & $<0.001$ \\
\hline Paralysis & $10.0 \%$ & $7.5 \%$ & 0.005 \\
\hline Transient Ischemic Attacks & $1.1 \%$ & $0.0 \%$ & $<0.001$ \\
\hline Family History of Stroke & $2.3 \%$ & $0.4 \%$ & $<0.001$ \\
\hline Acute but ill-defined Cerebrovascular Disease & $0.3 \%$ & $0.0 \%$ & 0.091 \\
\hline Epilepsy & $7.4 \%$ & $10.1 \%$ & $<0.001$ \\
\hline Other neurological disorders & $5.5 \%$ & $2.6 \%$ & $<0.001$ \\
\hline Depression & $10.7 \%$ & $13.2 \%$ & 0.007 \\
\hline Psychoses & $3.9 \%$ & $2.6 \%$ & 0.034 \\
\hline Alcohol abuse & $4.5 \%$ & $3.1 \%$ & 0.021 \\
\hline Drug Abuse & $3.1 \%$ & $1.8 \%$ & 0.011 \\
\hline Deficiency anemia & $14.7 \%$ & $12.8 \%$ & 0.063 \\
\hline
\end{tabular}




\section{Cureus}

$\begin{array}{llll}\text { Coagulopathy } & 5.5 \% & 4.0 \% & 0.025 \\ \text { Metastatic cancer } & 1.9 \% & 3.1 \% & 0.005 \\ \text { Solid Tumor without Metastasis } & 1.9 \% & 3.1 \% & 0.005 \\ \text { Diabetes, uncomplicated } & 29.8 \% & 26.0 \% & 0.005 \\ \text { Oral contraceptive use } & 0.1 \% & 0.4 \% & 0.009 \\ \text { Renal failure } & 16.3 \% & 11.5 \% & <0.001 \\ \text { Rhabdomyolysis } & 1.6 \% & 0.4 \% & <0.001 \\ \text { Fluid and electrolyte Disorders } & 27.6 \% & 20.3 \% & <0.001 \\ \text { Liver Disease } & 1.7 \% & 1.3 \% & 0.334 \\ \text { Obesity } & 11.4 \% & 10.6 \% & 0.356\end{array}$

\section{TABLE 1: Baseline characteristics of hospitalized acute ischemic stroke patients without versus with cocaine.}

Significant $P$-values $\leq 0.05$ at $95 \%$ confidence interval, variables were Agency for Healthcare Research and Quality (AHRQ) comorbidity measures.

Abbreviations: $\mathrm{AIS}=$ acute ischemic strokes, $\mathrm{SNF}=$ skilled nursing facility, INF= intermediate nursing facility, RA= rheumatoid arthritis, CVD = collagen vascular diseases.

\section{Multivariable risk factors for acute ischemic strokes hospitalization}

Table 2 shows different variables that were used in the multivariate logistic regression model to identify AIS risk factors requiring hospitalization. We found cocaine use to be the major risk factor for hospitalization. In-hospital mortality was also observed to be higher in cocaine cohort with the 95\% confidence interval (CI) 1.1-1.9 (OR=1.4, 95\% CI=1.1-1.9, p <0.003). A disposition to short-term hospitals $(\mathrm{OR}=2.6,95 \% \mathrm{CI}=2.1-3.3, \mathrm{p}<0.001)$ and home healthcare $(\mathrm{OR}=1.5,95 \% \mathrm{CI}=1.2-1.9, \mathrm{p}<0.001)$ was also significantly higher after adjusting for confounders. Personal history of sudden cardiac arrests $(\mathrm{OR}=7.9,95 \% \mathrm{CI}=3.1-20.1, \mathrm{p}<0.001)$ were significantly associated with cocaine use which could be another manifestation due to potential vasospasm [8]. Venous thromboembolism was observed to be linked with cocaine use $(\mathrm{OR}=1.5,95 \% \mathrm{CI}=1.0-2.1, \mathrm{p}<0.01)$, but less severely than vasculitis $(\mathrm{OR}=3.0,95 \% \mathrm{CI}=1.6-5.8$, $\mathrm{p}<0.001$ ) (Table 2).

\begin{tabular}{|c|c|c|c|c|}
\hline Variables & Odds Ratio & $95 \% \mathrm{Cl}$ & $99 \% \mathrm{Cl}$ & P-value* \\
\hline \multicolumn{5}{|c|}{ Weekend Admissions } \\
\hline Monday-Friday & Referent & Referent & Referent & \\
\hline Saturday-Sunday & 0.952 & $0.827-1.096$ & $0.791-1.145$ & 0.492 \\
\hline
\end{tabular}




\section{Cureus}

Routine

To Short-term Hospitals

Other (SNF, ICF, Another Facility)

Home Health Care

Against Medical Advice (AMA)

Died

Elective versus Non-elective Admissions

Non-elective

Elective

Indicator of Sex

Male

Female

Length of stay (cleaned)

1 to 3 days

4 to 6 days

7 to 9 days

10 to 12 days

$\geq 13$ days

Primary Expected Payer

Medicare

Medicaid

Private including $\mathrm{HMO}$

Self - Pay

Other

Race

White

Black

Hispanic

Other

Median Household Income Quartile on Patient's ZIP

$\begin{array}{llll}\text { Referent } & \text { Referent } & \text { Referent } & \\ 2.691 & 2.143-3.380 & 1.995-3.630 & <0.001 \\ 0.970 & 0.809-1.164 & 0.764-1.233 & 0.745 \\ 1.574 & 1.291-1.920 & 1.213-2.043 & <0.001 \\ 1.370 & 0.719-2.610 & 0.587-3.196 & 0.338 \\ 1.485 & 1.147-1.922 & 1.058-2.084 & 0.003\end{array}$

0.374

$0.238-0.588$

$0.206-0.678$

$<0.001$

Referent Referent Referent

Referent Referent Referent

0.966

$0.849-1.098$

$0.816-1.144$

0.597

Referent

Referent

Referent

0.776

$0.661-0.910$

$0.629-0.957$

0.002

1.074

$0.876-1.316$

$0.822-1.403$

0.493

1.159

$0.886-1.515$

$0.815-1.649$

0.281

1.252

$0.988-1.588$

$0.917-1.711$

0.063

1.311

$0.694-2.476$

$0.568-3.024$

0.405

1.757

$0.904-3.415$

$0.733-4.208$

0.097

1.048

$0.548-2.002$

$0.447-2.454$

0.888

0.635

$0.262-1.544$

$0.198-2.040$

0.317

Referent

Referent

Referent

$\begin{array}{llll}9.310 & 3.854-22.488 & 2.921-29.668 & <0.001 \\ 3.883 & 1.563-9.648 & 1.174-12.842 & 0.003 \\ 6.792 & 2.688-17.158 & 2.009-22.958 & <0.001 \\ \text { Referent } & \text { Referent } & \text { Referent } & \end{array}$

Referent Referent Referent 


\section{Cureus}

$\$ 1-\$ 39,999$

$\$ 40,000-\$ 50,999$

$\$ 51,000-\$ 65,999$

$\$ 66,000+$

Bed Size of Hospital

Small

Medium

Large

Location and Teaching Status of Hospital

Rural

Urban - non teaching

Urban - teaching

Control/ownership of Hospital

Government, non-federal

Private, non profit

Private, invest -own

Co -morbidities ${ }^{\#}$

Musculoskeletal

RA/CVD

Connective Tissue Disorder

Cardiovascular

Congestive Heart Failure

Atherosclerosis

AMI

Arrhythmia

Sudden Cardiac Death

SupraVentricular Premature Beats

Cardiac Circulatory Anomalies

Cardiomyopathy

Tachycardia

Heart Valve Disorders

$\begin{array}{llll}0.610 & 0.489-0.761 & 0.456-0.815 & <0.001 \\ 0.734 & 0.615-0.876 & 0.582-0.926 & <0.001 \\ & & & \\ 0.977 & 0.843-1.133 & 0.805-1.186 & 0.759\end{array}$

Referent Referent Referent

1.426

$1.225-1.662$

$1.167-1.743$

$<0.001$

1.158

$1.001-1.340$

$0.957-1.402$

0.048

Referent Referent

Referent

1.573

$1.244-1.989$

$1.156-2.141$

$<0.001$

1.061

$0.909-1.238$

$0.866-1.299$

0.452

Referent

Referent

Referent

0.198

$0.077-0.505$

$0.058-0.678$

$<0.001$

1.306

$0.944-1.805$

$0.853-1.999$

0.107

Referent Referent

Referent

1.464

$1.039-2.063$

$0.933-2.297$

0.029

0.800

$0.392-1.632$

0.313- 2.042

0.539

1.156

$0.971-1.376$

$0.919-1.453$

0.104

0.789

$0.681-0.913$

$0.651-0.956$

$<0.001$

0.841

$0.622-1.137$

$0.566-1.249$

0.259

2.744

$1.093-6.886$

$0.819-9.194$

0.032

7.950

$3.135-20.163$

$2.340-27.013$

$<0.001$

2.478

$1.009-6.085$

$0.761-8.070$

0.048

0.280

$0.149-0.525$

$0.122-0.640$

$<0.001$

1.085

$0.832-1.415$

$0.766-1.538$

0.546

0.867

$0.357-2.107$

$0.270-2.785$

0.753

0.679

$0.363-1.270$

$0.298-1.546$

0.226 


\section{Cureus}

Peripheral Vascular Disorders

Vasculitis

Hypertension

Elevated Cholesterol

Aortic and Peripheral Arterial Embolism or Thrombosis

Venous Thromboembolism

Respiratory

Chronic Pulmonary Disease

Pneumothorax (pleurisy)

Pulmonary Circulation Disorders

Neurological

Paralysis

Family History of Stroke

Meningitis

Migraine

Epilepsy

Other Neurological Disorders

Psychiatry

Depression

Psychoses

Alcohol abuse

Drug Abuse

Hemato-oncological

Deficiency Anemia

Chronic Blood Loss Anemia

Coagulopathy

Weight Loss

Metastatic Cancer

Solid Tumor without Metastasis

Lymphoma

Endocrinological

$\begin{array}{llll}1.200 & 0.989-1.456 & 0.931-1.547 & 0.065 \\ 3.077 & 1.609-5.886 & 1.312-7.217 & <0.001 \\ 0.982 & 0.846-1.139 & 0.807-1.194 & 0.807 \\ 0.642 & 0.564-0.731 & 0.541-0.761 & <0.001 \\ 0.682 & 0.278-1.670 & 0.210-2.214 & 0.402 \\ 1.518 & 1.097-2.100 & 0.991-2.326 & 0.012\end{array}$

0.895

$0.752-1.064$

$0.712-1.123$

0.207

0.944

$0.677-1.316$

$0.610-1.460$

0.733

1.133

$0.852-1.507$

$0.779-1.649$

0.391

0.695

$0.540-0.895$

$0.499-0.969$

0.005

0.270

$0.112-0.654$

$0.085-0.864$

0.004

1.951

$0.786-4.842$

$0.591-6.443$

0.150

1.157

$0.833-1.607$

$0.752-1.782$

0.383

1.563

$1.266-1.930$

$1.185-2.062$

$<0.001$

0.469

$0.320-0.687$

$0.284-0.775$

$<0.001$

1.486

$1.243-1.777$

$1.175-1.880$

$<0.001$

0.832

$0.575-1.205$

$0.512-1.354$

0.331

0.761

$0.535-1.082$

$0.479-1.208$

0.128

0.964

$0.603-1.541$

$0.521-1.786$

0.879

1.061

$0.878-1.281$

$0.827-1.360$

0.542

1.071

$0.437-2.626$

$0.330-3.480$

0.880

0.811

$0.593-1.109$

$0.538-1.224$

0.190

1.308

$0.986-1.736$

$0.902-1.898$

0.063

1.052

$0.722-1.533$

$0.642-1.725$

0.791

1.109

$0.764-1.610$

$0.680-1.810$

0.585

0.553

$0.228-1.343$

$0.173-1.774$

0.191 


\section{Cureus}

Diabetes, Uncomplicated

Diabetes with Chronic Complications

Renal

Acute Renal Failure

Rhabdomyolysis

Fluid and Electrolyte Disorders

Gastrointestinal

Obesity
Liver Disease

$\begin{array}{lll}1.278-1.852 & 1.205-1.964 & <0.001 \\ 0.092-0.544 & 0.070-0.718 & <0.001 \\ 0.518-0.725 & 0.491-0.764 & <0.001\end{array}$

$0.540-1.544$

$0.458-1.821$

0.734

\section{TABLE 2: Predictors of hospitalization in acute ischemic strokes (AIS) cocaine cohort versus acute ischemic strokes (AIS) on cocaine cohort by multivariate logistic regression.}

Significant $P$-value $\leq 0.05$ at $95 \%$ and $\leq 0.01$ at $99 \%$ confidence interval, variables are Agency for Healthcare Research and Quality (AHRQ) co-morbidity measures.

Abbreviations: SNF= skilled nursing facility, INF= intermediate nursing facility, RA= rheumatoid arthritis CVD= collagen vascular diseases, $\mathrm{Cl}=$ confidence interval, $\mathrm{HMO}=$ Health Maintenance Organization.

\section{Gender comparison of cocaine-associated mortality}

Table 3 shows the gender comparison in co-morbidities associated mortality in the cocaine cohort. Higher overall mortality due to cardiac (except arrhythmia and supraventricular premature beats) causes and acute renal failure was observed in males, whereas females had increased overall mortality owing to elevated cholesterol, heart valve disorders, vasculitis, epilepsy, arrhythmia, supraventricular premature beats (SVPB), peripheral arterial thromboembolism and heart valve disorders. A similar rate of mortalities between males and females was found due to events of elevated blood pressure without hypertension (which could be owing to incidental cocaine intake) and acute cerebrovascular disease.

\section{Comorbidities and predictors of mortality}

\section{Cocaine use}

Cardiomyopathy

Acute myocardial infarction

\begin{tabular}{|c|c|c|}
\hline \multicolumn{2}{|l|}{ Died } & \multirow{2}{*}{ P-value } \\
\hline Male & Female & \\
\hline $0.2 \%$ & $0.3 \%$ & 0.160 \\
\hline $11.9 \%$ & $9.0 \%$ & $<0.001$ \\
\hline $15.5 \%$ & $13.7 \%$ & $<0.001$ \\
\hline
\end{tabular}




\section{Cureus}

\begin{tabular}{|c|c|c|c|}
\hline Atherosclerosis & $36.0 \%$ & $27.4 \%$ & $<0.001$ \\
\hline Acute renal failure & $40.7 \%$ & $31.3 \%$ & $<0.001$ \\
\hline Arrhythmia & $0.2 \%$ & $0.4 \%$ & $<0.001$ \\
\hline Supraventricular Premature Beats & $0.1 \%$ & $0.2 \%$ & $<0.001$ \\
\hline Sudden cardiac death & $0.6 \%$ & $0.3 \%$ & $<0.001$ \\
\hline Cardiac and circulatory anomalies & $2.2 \%$ & $1.5 \%$ & $<0.001$ \\
\hline Transient ischemic attacks & $0.6 \%$ & $0.5 \%$ & $<0.001$ \\
\hline Tachycardia & $1.9 \%$ & $1.8 \%$ & $<0.001$ \\
\hline Elevated BP without hypertension & $0.1 \%$ & $0.1 \%$ & $<0.001$ \\
\hline Pneumothorax and pleurisy & $12.3 \%$ & $9.8 \%$ & $<0.001$ \\
\hline Bronchiolitis obliterans organizing pneumonia & $0.1 \%$ & $0.1 \%$ & $<0.001$ \\
\hline Rhabdomyolysis & $4.3 \%$ & $3.0 \%$ & $<0.001$ \\
\hline Elevated cholesterol and lipids & $35.1 \%$ & $35.3 \%$ & $<0.001$ \\
\hline Meningitis & $1.2 \%$ & $0.7 \%$ & $<0.001$ \\
\hline Migraine & $0.6 \%$ & $1.3 \%$ & $<0.001$ \\
\hline Sickle cell disease & $0.1 \%$ & $0.2 \%$ & $<0.001$ \\
\hline Oral contraceptive use & $0.0 \%$ & $0.1 \%$ & 0.472 \\
\hline Viral infection & $1.0 \%$ & $0.6 \%$ & $<0.001$ \\
\hline Heart valve disorder & $11.3 \%$ & $12.2 \%$ & $<0.001$ \\
\hline Vasculitis & $0.4 \%$ & $0.6 \%$ & $<0.001$ \\
\hline Connective tissue disorder & $0.2 \%$ & $1.1 \%$ & 0.107 \\
\hline Aortic, peripheral arterial thromboembolism & $1.6 \%$ & $2.0 \%$ & $<0.001$ \\
\hline Acute vascular insufficiency of intestine & $0.9 \%$ & $0.6 \%$ & $<0.001$ \\
\hline Epilepsy & $11.9 \%$ & $12.2 \%$ & $<0.001$ \\
\hline Family History Stroke (cerebrovascular) & $0.6 \%$ & $0.7 \%$ & $<0.001$ \\
\hline Acute but ill-defined cerebrovascular disease & $0.4 \%$ & $0.4 \%$ & $<0.001$ \\
\hline Drug induced headache & $0.0 \%$ & $0.0 \%$ & 0.071 \\
\hline
\end{tabular}

TABLE 3: Gender comparison in comorbidities associated mortality in acute ischemic stroke (AIS) cocaine cohort. 


\section{Multivariable predictors of mortality}

Table 4 shows the comparison of various comorbidity related mortality odds between cocaine and non-cocaine cohorts. Mortality odds owing to liver disease, metastatic cancer, cardiomyopathy, acute myocardial infarction, and epilepsy were increased in both non-cocaine and cocaine cohort. Whereas, increased odds of mortality in the non-cocaine cohort were observed due to coagulopathy, disorders of fluid and electrolyte, obesity, weight loss, solid tumor without metastasis, elevated cholesterol, pneumothorax and pleurisy and congestive heart failure. Effect on mortality due to other variables is shown in Table 4.

\begin{tabular}{|c|c|c|c|c|c|c|c|c|}
\hline \multirow[t]{2}{*}{ Variables } & \multicolumn{4}{|c|}{ No Cocaine } & \multicolumn{4}{|c|}{ Cocaine } \\
\hline & $\begin{array}{l}\text { Odds } \\
\text { ratio }\end{array}$ & $\begin{array}{l}99 \% \text { C } \\
\text { Interva }\end{array}$ & idence & $\begin{array}{l}\text { P- } \\
\text { value* }\end{array}$ & $\begin{array}{l}\text { Odds } \\
\text { ratio }\end{array}$ & $\begin{array}{l}99 \% \text { C } \\
\text { Interva }\end{array}$ & fidence & $\begin{array}{l}\text { P- } \\
\text { value* }\end{array}$ \\
\hline \multicolumn{9}{|l|}{ Co - morbidities $\#$} \\
\hline Deficiency anemias & 0.899 & 0.866 & 0.933 & $<0.001$ & 1.809 & 0.622 & 5.258 & 0.153 \\
\hline Congestive heart failure & 1.540 & 1.487 & 1.596 & $<0.001$ & 0.506 & 0.174 & 1.471 & 0.100 \\
\hline Chronic pulmonary disease & 1.125 & 1.085 & 1.166 & $<0.001$ & 1.078 & 0.431 & 2.694 & 0.833 \\
\hline Coagulopathy & 1.720 & 1.644 & 1.800 & $<0.001$ & 0.378 & 0.042 & 3.428 & 0.256 \\
\hline Depression & 0.751 & 0.713 & 0.791 & $<0.001$ & 0.191 & 0.048 & 0.753 & 0.002 \\
\hline Diabetes, uncomplicated & 0.891 & 0.862 & 0.921 & $<0.001$ & 0.364 & 0.156 & 0.850 & 0.002 \\
\hline Hypertension & 0.725 & 0.701 & 0.749 & $<0.001$ & 0.302 & 0.119 & 0.765 & 0.001 \\
\hline Hypothyroidism & 1.031 & 0.990 & 1.074 & 0.052 & 1.521 & 0.527 & 4.395 & 0.308 \\
\hline Liver disease & 1.210 & 1.107 & 1.322 & $<0.001$ & 12.608 & 1.255 & 126.656 & 0.005 \\
\hline Fluid and electrolyte disorders & 1.582 & 1.534 & 1.632 & $<0.001$ & 1.046 & 0.430 & 2.546 & 0.896 \\
\hline Metastatic cancer & 1.921 & 1.787 & 2.065 & $<0.001$ & 4.895 & 1.318 & 18.184 & 0.002 \\
\hline Other neurological disorders & 1.129 & 1.074 & 1.187 & $<0.001$ & 0.461 & 0.064 & 3.314 & 0.312 \\
\hline Obesity & 0.754 & 0.717 & 0.793 & $<0.001$ & 0.694 & 0.204 & 2.356 & 0.441 \\
\hline Paralysis & 1.494 & 1.437 & 1.553 & $<0.001$ & 0.929 & 0.269 & 3.207 & 0.879 \\
\hline Peripheral vascular disorders & 1.145 & 1.097 & 1.196 & $<0.001$ & 3.405 & 1.296 & 8.947 & 0.001 \\
\hline Pulmonary circulation disorders & 1.268 & 1.195 & 1.345 & $<0.001$ & 0.295 & 0.075 & 1.157 & 0.021 \\
\hline Renal failure & 1.077 & 1.037 & 1.119 & $<0.001$ & 0.775 & 0.222 & 2.702 & 0.599 \\
\hline $\begin{array}{l}\text { Solid tumor without } \\
\text { metastasis }\end{array}$ & 1.279 & 1.174 & 1.394 & $<0.001$ & 0.534 & 0.075 & 3.785 & 0.409 \\
\hline Weight loss & 1.263 & 1.206 & 1.323 & $<0.001$ & 0.959 & 0.296 & 3.107 & 0.927 \\
\hline Cardiomyopathy & 1.076 & 1.024 & 1.132 & $<0.001$ & 3.008 & 0.844 & 10.722 & 0.026 \\
\hline
\end{tabular}




\section{Cureus}

$\begin{array}{lcccccccc}\text { Acute myocardial infarction } & 2.431 & 2.323 & 2.544 & <0.001 & 7.820 & 2.173 & 28.138 & <0.001 \\ \text { Atherosclerosis } & 1.071 & 1.037 & 1.106 & <0.001 & 0.844 & 0.371 & 1.918 & 0.594 \\ \text { Tachycardia } & 1.950 & 1.746 & 2.179 & <0.001 & 2.133 & 0.131 & 34.728 & 0.484 \\ \text { Elevated Cholesterol and lipid } & 0.558 & 0.541 & 0.575 & <0.001 & 0.815 & 0.325 & 2.042 & 0.566 \\ \text { Pneumothorax and pleurisy } & 1.622 & 1.542 & 1.706 & <0.001 & 0.263 & 0.048 & 1.449 & 0.044 \\ \text { Epilepsy } & 1.479 & 1.412 & 1.550 & <0.001 & 9.322 & 3.721 & 23.355 & <0.001\end{array}$

\section{TABLE 4: Multivariate predictors of the mortality in acute ischemic stroke patients without cocaine use versus with cocaine use}

Significant P-values $\leq 0.05$ at $95 \%$ and $\leq 0.01$ at $99 \%$ confidence interval, variables are Agency for Healthcare Research and Quality (AHRQ) co-morbidity measures.

\section{Discussion}

The current study found $96.5 \%$ of the AIS cocaine cohort was of the age group above 45 years with age ranging from 18 years to 85 years and above. In the age group of 85 years and above, the prevalence of AIS within the cocaine group surpassed the non-cocaine users. A plausible explanation could be that the cumulative effect of traditional risk factors, along with the longterm accumulation of chronic cocaine effect makes such population more vulnerable towards the risk of stroke [2]. The frequency of hospitalization was high among the urban hospitals set up.

A majority of the AIS patients visited the private, nonprofit hospitals. Among these, the odds of ones with cocaine use visiting the government, non-federal hospital were significantly low. The nature of the admission was nonelective understandable for most of the AIS cohort and within this cohort; it was significantly higher among the cocaine users. It could be because most of the patients are chronic cocaine abusers rather than acute. This finding is also justifiable from the older age group pattern of the study subject which is prone to the cumulative effect of the cocaine rather than acute features [2]. Odds of hospitalization among the whites were higher compared to the blacks and Hispanics in AIS cocaine cohort versus AIS non-cocaine cohort.

The mortality was found higher in blacks as compared to whites and Hispanic $(21.4 \%$ versus $8.6 \%$ versus $11.1 \%, \mathrm{P}=0.004$ respectively). Analyzing the disposition of the patients, the shortterm hospitals stay and death was significantly higher among the cocaine users. The associated higher comorbidities could be a possible explanation for such disposition in cocaine users as compared to the non-cocaine group. This finding is a serious concern because such patients could lead to a significant burden on the healthcare infrastructure. With increasing median household income, the frequency of hospitalization significantly increased among the cocaine users suggesting that the ones with low income and living in a poverty have a lower risk of using cocaine. Another reason could be that socioeconomic status is the poor predictor of the stroke among the cocaine users. Despite having high median household income among the cocaine users, their hospitalization was significantly elevated in the small and medium-sized hospitals. This could be due to the acute nature of the condition among these subjects, requiring urgent admission to any of the nearby facility.

The family history of the stroke was higher among the non-cocaine users as compared to the 
cocaine users suggesting that the usual mechanism of stroke development is not applicable to the cocaine user. Cocaine users quite commonly bear the traditional cardiovascular risk factors [2]. Sudden cardiac death, paroxysmal supraventricular tachycardia (PSVT), vasculitis and venous thromboembolism take higher odds of hospitalization.

Odds of hospitalization due to the paralysis were significantly higher among the non-cocaine users while seizures were high among the cocaine users. The frequency of depression was significantly higher among the cocaine abusers signifying the high morbidity among such populations. The incidence of diabetes and congestive heart failure (CHF) was severely high among the cocaine users, while the frequency of hypertension was quite similar to the other studies [2].

There was a significantly higher rate of valvular heart disease and venous thromboembolism among the cocaine users, suggesting of emboli as the major risk for stroke among these populations as compared to the non-cocaine users [15-17]. We reported the higher mortality among the cocaine users as compared to the non-cocaine users [18].

The older age of our study population could be a plausible reason, as studies with the young demographic and mild strokes have reported overall low mortality [19]. When we looked for the multivariate predictors of death in the AIS patients without cocaine use and with cocaine use, we found that epilepsy, peripheral vascular disorders, acute myocardial infarction, cardiomyopathy, tachycardia, metastatic cancer and liver diseases were associated with higher odds of mortality among cocaine users as compared to the non-cocaine users.

Hypertension and diabetes were not found to be associated with the excess mortality in an AIS cocaine cohort compared to the AIS non-cocaine cohort. Several postulated mechanisms for cocaine-induced ischemic stroke has been suggested [20-23]. Among these, the cardioembolic ischemic strokes and cardiac deaths due to chronic cocaine use have been proposed to be prominent [17, 24].

\section{Study limitations}

This study has undeniable limitations because of the NIS database which might have coding errors in terms of determining the diagnosis, comorbidities, and complications. Due to the inherent nature of large hospital's database, it may over or underestimate AIS, cocaine use, comorbidities and other clinically relevant variables based on ICD-9 CM codes. This study also lacked variables such as medications and other treatment options related to AIS.

This database does not mention about the cause of death, so we cannot differentiate between in-hospital events and cause of death. It might be possible to have a selection bias in this study because of a retrospective population study. Due to large data size and getting national estimates using discharge weight as provided by NIS database, we could overcome these limitations.

\section{Conclusions}

To our knowledge, this is one of the very few studies demonstrating the effects of cocaine use on stroke using the nationally representative data source. Our results displaying the amplitude of the mortality in an AIS-cocaine cohort raised the question whether to consider cocaine as a risk factor in all AIS patients or not. Further research is warranted to evaluate the pathogenesis and health care burden due to cocaine-induced stroke.

\section{Appendices}




\section{Cureus}

Table 5 has the list of ICD-9-CM codes for suspected risk factors of AIS, comorbidities, inhospital procedures and outcomes.

\begin{tabular}{|c|c|c|}
\hline Risk Factors/ Co-morbidity & Source & Codes \\
\hline Acute ischemic stroke & ICD-9 & $\begin{array}{l}\text { 433.01, 433.10, 433.11, 433.21, 433.31, 433.81, 433.91, } \\
434.00,434.01,434.11,434.91,436\end{array}$ \\
\hline Cocaine & ICD - 9 & $304.20,304.21,304.22,305.60305 .61,305.62$ \\
\hline Acute myocardial infarction & CCS & 100 \\
\hline Peri-; endo-; and myocarditis, cardiomyopathy & CCS & 97 \\
\hline Sudden cardiac death & ICD - 9 & V12.53 \\
\hline Arrhythmias & ICD - 9 & 427.9 \\
\hline Supraventricular premature beats & ICD - 9 & 427.61 \\
\hline Tachycardia, unspecified & ICD - 9 & 785.0 \\
\hline $\begin{array}{l}\text { Elevated blood pressure reading without } \\
\text { diagnosis of hypertension }\end{array}$ & ICD - 9 & 796.2 \\
\hline Rhabdomyolysis & ICD - 9 & 728.88 \\
\hline Acute and unspecified renal failure & CCS & 157 \\
\hline Bronchiolitis Obliterans organizing pneumonia & ICD - 9 & 516.8 \\
\hline Pleurisy; pneumothorax; pulmonary collapse & CCS & 130 \\
\hline Epilepsy & CCS & 83 \\
\hline $\begin{array}{l}\text { Drug induced headache, not elsewhere } \\
\text { classified }\end{array}$ & ICD - 9 & 339.3 \\
\hline Family Hx Stroke (cerebrovascular) & ICD - 9 & V17.1 \\
\hline Acute vascular insufficiency of intestine & ICD - 9 & 557.0 \\
\hline $\begin{array}{l}\text { Aortic and peripheral arterial embolism or } \\
\text { thrombosis }\end{array}$ & CCS & 116 \\
\hline Unspecified venous complication & ICD - 9 & 671.9 \\
\hline Venous thrombosis and embolism & ICD - 9 & V12.51 \\
\hline Connective tissue diseases & CCS & 210 \\
\hline Vasculitis & ICD - 9 & $447.6 ; 446.0-446.9$ \\
\hline Meningitis & CCS & 76 \\
\hline Cardiac and circulatory anomalies & CCS & 213 \\
\hline Elevated cholesterol and lipids & CCS & 53 \\
\hline
\end{tabular}




\section{Cureus}

$\begin{array}{llc}\text { Migraine } & \text { CCS } & 84 \\ \text { Sickle cell disease } & \text { CCS } & 61 \\ \text { Viral infection } & \text { CCS } & 7 \\ \text { Heart valve disorder } & \text { CCS } & 96 \\ \text { Atherosclerosis } & \text { CCS } & 114 \\ \text { Acute but ill-defined cerebrovascular disease } & \text { CCS } & 109 \\ \text { Transient ischemic attack } & \text { CCS } & 112 \\ \text { Oral contraceptive use } & \text { CCS } & 176\end{array}$

TABLE 5: International Classification of Disease, Ninth Revision (ICD-9) codes and the Clinical Classifications Software (CCS) codes used to identify co-morbidities, inhospital procedures and complications.

Abbreviations: ICD-9-CM= International Classification of Diseases, Ninth Revision, Clinical Modification; CCS= Clinical Classification Software.

\section{Additional Information}

\section{Disclosures}

Human subjects: All authors have confirmed that this study did not involve human participants or tissue. Animal subjects: All authors have confirmed that this study did not involve animal subjects or tissue. Conflicts of interest: In compliance with the ICMJE uniform disclosure form, all authors declare the following: Payment/services info: All authors have declared that no financial support was received from any organization for the submitted work. Financial relationships: All authors have declared that they have no financial relationships at present or within the previous three years with any organizations that might have an interest in the submitted work. Other relationships: All authors have declared that there are no other relationships or activities that could appear to have influenced the submitted work.

\section{References}

1. Zimmerman JL: Cocaine intoxication. Crit Care Clin. 2012, 28:517-526. 10.1016/j.ccc.2012.07.003

2. Bhattacharya P, Taraman S, Shankar L, et al.: Clinical profiles, complications, and disability in cocaine-related ischemic stroke. J Stroke Cerebrovasc Dis. 2011, 20:443-449. 10.1016/j.jstrokecerebrovasdis.2010.02.017

3. MacEwen C, Ward M, Buchan A: A case of cocaine-induced basilar artery thrombosis . Nat Rev Neurol. 2008, 4:622-626. 10.1038/ncpneuro0879

4. Sordo L, Indave BI, Barrio G, et al.: Cocaine use and risk of stroke: A systematic review . Drug Alcohol Depend. 2014, 142:1-13. 10.1016/j.drugalcdep.2014.06.041

5. U.S. DEPARTMENT OF HEALTH AND HUMAN SERVICES : Results from the 2012 National Survey on Drug Use and Health: Summary of National Findings. NSDUH Series H-48, HHS Publication No. (SMA) 13-4795. Rockville,MD; 2013.

6. Chait R, Fahmy S, Caceres J: Cocaine abuse in older adults: An underscreened cohort. J Am Geriatr Soc. 2010, 58:391-392. 10.1111/j.1532-5415.2009.02697.x

7. Calkins, RF: Drug abuse trends in Detroit/Wayne County and Michigan in Epidemiologic 
Trends in Drug Abuse, Vol II: Proceedings of the Community Epidemiology Work Group.. National Institute on Drug Abuse. Bethesda, MD; 2002. 66-77.

8. Bodmer M, Enzler F, Liakoni E, et al.: Acute cocaine-related health problems in patients presenting to an urban emergency department in Switzerland: A case series. BMC Res Notes. 2014, 7:1-7. 10.1186/1756-0500-7-173

9. Barrio G, Molist G, de la Fuente L, et al.: Mortality in a cohort of young primary cocaine users: controlling the effect of the riskiest drug-use behaviors. Addict Behav. 2013, 38:1601-1604. 10.1016/j.addbeh.2012.10.007

10. Degenhardt L, Singleton J, Calabria B, et al: Mortality among cocaine users: A systematic review of cohort studies. Drug Alcohol Depend. 2011, 113:88-95. 10.1016/j.drugalcdep.2010.07.026

11. Murray CJ, Vos T, Lozano R, et al: Disability-adjusted life years (DALYs) for 291 diseases and injuries in 21 regions, 1990-2010: a systematic analysis for the Global Burden of Disease study 2010. The Lancet. 2012, 380:2197-2223. 10.1016/S0140-6736(12)61689-4

12. National Institute of Neurological Disorders and Stroke. Final Report of the Stroke Progress Review Group-January 2012. (2013). Accessed: February 21: http://www.ninds.nih.gov/find_people/groups/stroke_prg/01-2012-stroke-prg-report.htm..

13. Introduction to the Nationwide Inpatient Sample (NIS). Agency for Healthcare Research and Quality. (2017). Accessed: July, 11: https://www.hcup-us.ahrq.gov/nisoverview.jsp.

14. Westover AN, McBride S, Haley RW: Stroke in young adults who abuse amphetamines or cocaine: a population-based study of hospitalized patients. Arch Gen Psychiatry. 2007, 64:495-502. 10.1001/archpsyc.64.4.495

15. Treadwell SD, Robinson TG: Cocaine use and stroke. Postgrad Med J. 2007, 83:389-394. 10.1136/pgmj.2006.055970

16. Nunez BD, Miao L, Ross JN, et al.: Effects of cocaine on carotid vascular reactivity in swine after balloon vascular injury. Stroke. 1994, 25:631-638. 10.1161/01.STR.25.3.631

17. Sloan MA, Mattioni TA: Concurrent myocardial and cerebral infarctions after intranasal cocaine use. Stroke. 1992, 23:427-30. 10.1161/01.STR.23.3.427

18. Giraldo EA, Taqi MA, Vaidean GD: A case-control study of stroke risk factors and outcomes in African American stroke patients with and without crack-cocaine abuse. Neurocrit Care. 2012, 16:273-279. 10.1007/s12028-010-9410-X

19. Thom T, Haase N, Rosamond W, et al: Heart disease and stroke statistics-2006 update: A report from the American Heart Association Statistics Committee and Stroke Statistics Subcommittee. Circulation. 2006, 113:85-151. 10.1161/CIRCULATIONAHA.105.171600

20. Pavarin R, Lugoboni F, Mathewson S, et al.: Cocaine-related medical and trauma problems: A consecutive series of 743 patients from a multicentre study in Italy. Eur J Emerg Med. 2011, 18:208-214. 10.1097/MEJ.0b013e3283440f25

21. Lange RA, Hillis LD: Cardiovascular complications of cocaine use . New Engl J Med. 2001, 345:351-358. 10.1056/NEJM200108023450507

22. Morales Vidal SG, Hornik A, Morgan C: Cocaine induced hippocampi infarction. BMJ Case Rep. 2012, 10.1136/bcr.03.2012.5998

23. Hollander JE, Hoffman RS: Cocaine-induced myocardial infarction: An analysis and review of the literature. J Emerg Med. 1992, 10:169-177. 10.1016/0736-4679(92)90212-C

24. Karch SB, Billingham ME: Coronary artery and peripheral vascular disease in cocaine users . Coronary Artery Disease. 1995, 6:220-225. 